\title{
Diferencia en la resiliencia según género y nivel socioeconómico en adolescentes
}

\author{
Rodolfo Prado Álvarez \& Mónica del Águila Chávez \\ Universidad Nacional Federico Villarreal
}

Lima - Perú

Se investigó si existían diferencias significativas en las áreas de resiliencia respecto del género y el nivel socioeconómico en adolescentes de 12 a 14 años, en tres colegios considerados típicos para estos estratos. El diseño fue descriptivocomparativo, aplicando la Escala de Resiliencia para Adolescentes (ERA), validado y estandarizado por Prado y Del Águila (2000). Con respecto al género se encontró que no hay diferencias significativas en las puntuaciones totales de resiliencia, pero sí se hallaron diferencias significativas en el área de interacción a favor del género femenino. En cuanto a los niveles socioeconómicos no se encontraron diferencias significativas en las puntuaciones totales; sin embargo, se hallaron diferencias significativas en las áreas de interacción y creatividad a favor del estrato bajo, $e$ iniciativa a favor del nivel alto.

\section{Resiliencia / adolescencia / género y nivel socioeconómico}

\section{Gender and socioeconomic level differences in resilience in adolescents}

We investigated if there were significant differences in resilience areas about gender and social status in adolescences from 12 to 14 years old, in three schools of Lima consideres typical to this 3 levels (high-medium-low). The design was comparative descriptive and was applied the Resilience Scale for Adolescences (ERA) which was built, validity and standardized by Prado and Del Águila (2000) bases on wollin and wollin theory. Respecting with the gender, we found that there wasn't significant difference in the total Resilience punctuation, but there were significant differences in the Interaction Area in favor of the female about social status, we didn't find significant difference in the total punctuation; eventhought there were significant differences in Interaction Area and Creativity Area in favor of the lowest status, and Iniciative Area in favor to the highest status.

Resilience / adolescences / gender / social status

Correo electrónico: rodolfoprado@hotmail.com

Persona 6, 2003, 179-196 


\section{INTRODUCCIÓN}

Las investigaciones sobre resiliencia, en los últimos años, están dirigidas principalmente en dos sentidos: riesgo y protección. Estos dos factores se han confundido con resiliencia, como lo advierte Grotberg en su ponencia "Nuevas tendencias en resiliencia", leída en el Seminario Internacional de Resiliencia, realizado en Lima, en octubre del 2000, quien también toma en cuenta la conexión que existe entre resiliencia, desarrollo y crecimiento humano, proponiendo que la resiliencia y los niveles socioeconómicos no están relacionados. Estas hipótesis de trabajo, realizadas desde la perspectiva de la investigación norteamericana, ameritan confrontarlas con la realidad nacional, tomando en cuenta los diversos enfoques que -desde las teorías del desarrollo- se hacen sobre la adolescencia.

Autores como Maddaleno (1994) afirman que los adolescentes tienen más riesgos de padecer crisis de distinto orden que otras etapas evolutivas, sobre todo porque están expuestos a conductas de riesgo a corto, mediano y largo plazos en distintos niveles, como sexuales, de maduración, socioeconómicos, patología física y psíquica. Dryfoos (1990) hace referencia a un marco teórico sobre programas de prevención para adolescentes, que pone énfasis en los factores de riesgo y en determinantes familiares, los que devienen del consumo de drogas, de la educación y de la cultura en general.
Rutter (1992) define la resiliencia como el conjunto de características de los niños y adolescentes que no tienen problemas de conducta o emocionales, pese a que han sido sometidos a experiencias de estrés, asociadas al daño y que, sin embargo configuran respuestas de resistencia individual y habilidad para rebotar y manejar la realidad adversa, saliendo fortalecidos de ésta. Establece que hay tres fuentes de resiliencia: el ambiente social facilitador, la fuerza psíquica interna y las habilidades sociales en el manejo de situaciones y competencia en resolución.

Krauskopf (1995) propone que la adversidad fortalece más que la no exposición al peligro, destaca que la sobreprotección vulnera la capacidad de respuesta a la adversidad, enfrentar la adversidad permite a los seres humanos que la padecen, alcanzar niveles de competencia y salud. La gente necesita tener oportunidades para generar respuestas resilientes. Define la resiliencia como una dimensión innata de factores constitucionales y biopsicológicos personales que se estructuran a partir de la adversidad.

Rutter (1992) establece diferencias de enfoque en el tratamiento de la resiliencia en la adolescencia y la juventud. La globalización, afirma, ha roto la homogeneidad de las culturas, concepto alrededor del cual gira la formación de la identidad de los jóvenes.

Sin embargo, nosotros no pensamos lo mismo, la globalización es un fenó- 
meno socioeconómico que intenta dar reglas de juego para todos, sobre la base de la ilusión de la igualdad, aunque es cierto que en nuestra cultura, por lo menos a la que calificamos, entre otros aspectos, de imitadora, los adolescentes tratan de imitar otras culturas de supuesta calidad mejorada, que la televisión, la internet y la masificación de la información apenas contribuye al conocimiento de cómo se actúa en otras latitudes. La puesta en escena de la tarea de identificación, es uno de los problemas que intentamos estudiar en esta y otras investigaciones, es decir, la forma como se procesa la globalización y si es verdad que los jóvenes logran elaborar su identidad a partir de esquemas diferentes de los suyos, o es que estos factores referenciales son factores de riesgo que juegan a favor de la crisis adolescente, y por ende favorecen aspectos resilientes.

Una de las tesis que fundamentan el trabajo con adolescentes parte de los conceptos en los que se basa la psicología del desarrollo, dando prioridad al estudio de la niñez para lograr un adulto sano, en desmedro de un enfoque centrado en la adolescencia en pro de una vejez sana. Es posible desarrollar el concepto de tiempo funcional, biológico, psicológico, social y legal, explicando cómo es que algunos tiempos están desfasados en la cultura y no se corresponden necesariamente, brindando una hipótesis enriquecedora a favor de nuestra tesis, en el sentido de estable- cer un concepto del adolescente con mayores responsabilidades, en lo biológico, psicológico y sociocultural, y no necesariamente un período de desarrollo desde la crisis.

Anthony (1983) establece que en la actualidad la brecha generacional entre los progenitores y sus hijos adolescentes es tan grande que casi han renunciado a tomar decisiones respecto de ellos. Obiols (1994) propone la tesis de que en el mundo posmoderno, la adolescencia se establece como modelo social y a partir de allí se "adolescentiza" la sociedad. Estos autores van más allá, proponiendo considerar a la adolescencia no como una etapa del ciclo vital sino como un modo de ser; el adolescente así criado puede ser un adulto más sano.

En cuanto al tratamiento del género, Burín (1998) afirma que las culturas establecen, con sus diferencias de enfoque, la moral materna que supone la subjetividad domesticada con características psíquicas de sujeción y receptividad; se construye un ideal maternal que las mujeres interiorizan con subjetividad.

La teoría de identidad de género propone la hipótesis de las identificaciones de roles sobre los modelos identificatorios familiares. Las mujeres desarrollan el concepto de identidad corporal y los hombres la identidad posicional respecto del rol masculino. Horney (1980) sostiene que el desprecio masculino a las mujeres y su devaluación proviene del temor masculino 
ante la omnipotencia materna en los estadios tempranos de la vida.

Prado (2000), en su investigación sobre estructura y funcionamiento familiar en adolescentes resilientes, efectuada en el cono norte de Lima, encuentra que respecto de la estructura familiar no hay diferencias significativas entre resilientes y no resilientes, y donde los resilientes presentan mayor número de categorías familiares. Y respecto del funcionamiento familiar existen diferencias significativas en el área de resolución de problemas a favor de los no resilientes.

Es de notar que se determinó que en la distribución por sexo en la población, en el grupo no resiliente la mayoría son mujeres, lo que nos lleva a investigar si el género es una variable que hay que tomar en cuenta en la determinación de la resiliencia.

Baldwin (1982), en investigaciones indirectas relacionadas con niños y familias resistentes al estrés, distingue entre niños resistentes al estrés y familias resistentes al estrés. También afirma que la clase social es una importante variable de riesgo, la cual no incide directamente en el niño; sus efectos están mediados por variables proximales, como quién es el que cuida al niño.

Las familias que viven en alto riesgo operan en un contexto diferente de aquellas que viven en bajo riesgo. Las familias de alto riesgo exitosas eran más restrictivas y autoritarias en su política de crianza y más vigilantes en el monitoreo de la obediencia de la política familiar de parte de los niños con familias exitosas de bajo riesgo.

Dolto (1990) afirma que la adolescencia ya no es considerada como una crisis sino como un estado, pero no en todos los adolescentes y jóvenes, sino en aquellos que pertenecen a sectores socioeconómicos bajos de la población, su entrada en la adultez es brusca y rápida, ya sea por su necesidad de trabajar, por embarazos prematuros $\mathrm{y}$ otros factores, en cambio en los estratos medios urbanos, la adolescencia ya no es un pasaje sino un producto nuevo, y que aun en la sexualidad tienen sus códigos propios.

Meler (1992) afirma que las nuevas prácticas de la vida vinculan a los seres humanos hombres y mujeres desde la subjetividad, y que los vínculos tienden a caracterizarse como transicionales, con conflictos derivados de la coexistencia de las expectativas contradictorias. Los niños y adolescentes del futuro tienen diversos conflictos por resolver, como los mecanismos identificatorios que toman como base a los abuelos, los padres, los congéneres y otras culturas de referencia en la globalización; por todo ello es necesario proponer estudios culturales que establezcan representatividades culturales.

Barbera (1998) afirma que el género se concibe como un conjunto de procesos de naturaleza biopsicosocial, determinado por la vinculación sexo-género, una categorización social normati- 
va, una construcción subjetiva, un proceso psicológico, como un sistema de clasificación y como un sistema dinámico interactivo.

Por todo lo explicado, nosotros afirmamos que, desde la resiliencia, es necesario conocer cuáles serían las diferencias significativas en el género, indagando acerca de los procesos estructurales de acuerdo con el esquema de Wolin y Wolin (1993) que se den en los adolescentes, hombres y mujeres, para poder establecer no solo un aspecto comparativo descriptivo, sino que nos sirva como elemento identificatorio que nos permita establecer los factores intervinientes en la formación de la resiliencia, y así generar estrategias de mejoramiento en la educación y en el medio comunitario, y nos permita desarrollar la tesis de que la adolescencia es un estado al que la psicología del desarrollo debería cambiar de enfoque.

Serrano (1995), en su investigación sobre la salud integral de los adolescentes y los jóvenes, su promoción y su cuidado, propone el enfoque contextual tomando el modelo económico y social elegido en los países que señalen rumbos y opciones en el espacio y el tiempo, establece los factores de riesgo en la familia, vecindario, comunidad, propiciando la participación de los jóvenes dentro de una estrategia interdisciplinaria, en la que hay que ir al encuentro del joven en sus propios ambientes, con un enfoque de riesgo que toma el concepto resiliencia, es decir, la capa- cidad de reacción o inmunidad, activando defensas protectoras, como mecanismos personales, a través de la familia y en instancias extrafamiliares, como organizaciones comunitarias juveniles.

Friedman (1998) desarrolla una metodología de estudio interdisciplinario e interinstitucional para el estudio de la salud del adolescente, en el marco de la OMS, planifica y promueve la investigación de la conducta y actitudes de los jóvenes, desarrollando técnicas de investigación en varios modelos: el enfoque cuadrícula, la técnica de investigación narrativa, técnicas de orientación, esquema del guardabarrera, interacción entre el usuario y el sistema y la dramatización. Los métodos se han empleado en diferentes sectores, lográndose el objetivo de estudiar los factores de riesgo y promover la salud desde la interacción con adultos, niños, psicólogos, dirigentes, etc.

Del estudio socioeconómico en la adolescencia, extraemos algunos conceptos como el de pobreza. Conceptualizaciones referidas a ésta las encontramos en Contreras (1998). La pobreza es explicada por causas individuales, cuyas características son: bajo nivel educativo, inteligencia poco desarrollada, falta de conocimientos, salud deficiente, discapacidad, orientación marginal laboral. También desde la inequidad en las oportunidades, inadecuadas demandas globales de trabajo y bajo índice nacional de ingreso. 
La cultura de la pobreza está explicada, pues, desde un enfoque estructural de ésta, que tiene como base las estructuras económicas y sociales existentes y un momento histórico determinado.

La resiliencia y las características socioeconómicas desde las perspectivas psicosociales, se refieren a la capacidad que tienen los individuos para resistir, sobreponerse y salir adelante, después de haber sido empobrecidos económicamente, y haber sufrido daños graves en la vida psíquica, moral y social. La resiliencia indica que la superación de la pobreza es posible, pero todo ello necesita de la capacidad que tiene el ser humano para resistir. La resiliencia cuenta con una base fundamental para responder a la pregunta acerca de si la pobreza es superable, y nosotros nos preguntamos si existen diferencias significativas en los adolescentes de 12 a 14 años en distintas clases sociales, tomando en cuenta los factores de resiliencia que determina el mandala de Wolin.

En los estudios sobre políticas sociales en el Perú que realiza Portocarrero (2000), establece varias hipótesis sobre heterogeneidad de la pobreza en la multidimensionalidad que han focalizado las políticas de los programas sociales. Establecen diferencias conceptuales entre pobreza y exclusión social, entendiendo a la pobreza no solamente en términos de bajos ingresos, necesidades básicas insatisfechas y oportunidades para alcanzar bienes básicos, si- no aspectos que refieren a dimensiones culturales de autoimagen y percepciones sobre carencias y posibilidades.

En cuanto a la exclusión se establece que es un concepto que va más allá de la pobreza. Se trata de las personas que están excluidas de la sociedad, implica no tener beneficios ni responsabilidades como ciudadanos. La exclusión social no tiene como base solamente un contexto económico, también establecen referencias étnicas, físicas, sexuales, de edad, empleo. Como se puede advertir, hay una diferencia entre ser pobre y ser excluido, las personas pueden ser pobres sin estar socialmente excluidas, y viceversa.

Tal es la dimensión del problema en el Perú que será necesario establecer, en cada clase, las características que determinen su nivel de pobreza y o de exclusión, nosotros - para efectos de la investigación- sólo abordaremos el concepto de pobreza tal cual la define Aramburu (1998), en el que se indican valores de estratificación económica, de bienes y servicios, educación en medios urbanos, cuya referencia de la muestra se realiza fundamentalmente en Lima. Las variables que tomaremos en cuenta se refieren a ingreso, empleo, vivienda, servicios básicos, bienes de consumo.

Del trabajo realizado por distintos investigadores, podemos apreciar que el estudio de la resiliencia en adolescentes se ha efectuado tomando en cuenta criterios educativos, distintas aprecia- 
ciones del concepto adolescencia y las redefiniciones al respecto, los conceptos de pobreza y diversos estudios referidos a la estratificación socioeconómica del país, pero no se han efectuado estudios referidos al género y a los niveles socioeconómicos, por todo ello nuestra investigación deberá establecer si existen diferencias significativas en adolescentes de 12 a 14 años con respecto al género y nivel socioeconómico, considerando los siete aspectos resilientes de Wolin.

\section{MATERIALES Y MÉTOdOS}

El diseño de investigación fue descriptivo-comparativo.

\section{Población y muestra}

La población investigada estuvo constituida por adolescentes de 12 a 14 años de tres centros educativos, correspondientes a las clases alta, media y baja de la ciudad de Lima.

Los centros educativos considerados típicos para estos estratos fueron los colegios Villa Caritas y San Pedro, del distrito de La Molina; Hans Christian Andersen, del distrito de Surco, y Fe y Alegría $\mathrm{N}^{\circ}$ 3, del distrito de San Juan de Miraflores. La población correspondió a 240 estudiantes.

La muestra estuvo conformada por 155 estudiantes del segundo año de los colegios antes mencionados.
Tabla No 1

Distribución de la muestra según género

\begin{tabular}{lcc}
\hline Género & Frecuencia & Porcentaje \\
\hline Femenino & 80 & 51,6 \\
Masculino & 75 & 48,4 \\
Total & 155 & 100 \\
\hline
\end{tabular}

La tabla 1 indica el número y porcentaje de la muestra por género.

Tabla $\mathrm{N}^{\circ} 2$

Distribución de la muestra según clase social

\begin{tabular}{lcc}
\hline Género & Frecuencia & Porcentaje \\
\hline Baja & 67 & 43,2 \\
Media & 46 & 29,7 \\
Alta & 42 & 27,1 \\
Total & 155 & 100 \\
\hline
\end{tabular}

La tabla 2 indica el número y el porcentaje de los niveles socioeconómicos de la muestra.

\section{Criterios de inclusión y exclusión}

Los criterios de inclusión que se tomaron en cuenta en la muestra fueron:

- Edad: 12 a 14 años.

- Clase social: baja, media y alta.

- Nivel de estudios: segundo año de secundaria.

- Género: masculino y femenino.

Los criterios de exclusión fueron:

- Edad: menores de 12 años y mayores de 14.

- Nivel de estudios: primaria; primero, tercero, cuarto y quinto de secundaria. 


\section{Instrumentos}

Se utilizó la Escala de Resiliencia para Adolescentes (ERA) que evalúa las siete áreas del mandala de Wolin: Insight, Creatividad, Interacción, Iniciativa, Moralidad, Humor e Independencia, la que consta de 34 ítems, que presentaron una validez en la correlación de Pearson con una consistencia interna de .0311 a 0.5083 y una confiabilidad por consistencia interna Alpha Cronbach con un coeficiente de 0.8629 , lo cual indica que es altamente significativo. El instrumento fue construido por Prado y Del Águila el año 2000.

\section{VALIDEZ Y CONFIABILIDAD DE LA ESCALA DE RESILIENCIA PARA \\ ADOLESCENTES}

En primer lugar se realizó un análisis de correlación ítem-test para verificar la discriminación de los ítems, para lo cual se empleó el coeficiente de correlación de Pearson (r).

Los datos se presentan en la tabla 3, donde se puede apreciar que todos los ítems presentan coeficientes superiores a 0.20 , el menor de todos es 0.2014 (p $<.05)$ y el máximo $0.5083(\mathrm{p}<.001)$, lo que indica que todos los ítems contribuyen a la medición de la resiliencia y por lo tanto aportan a la validez del instrumento.

Tabla N $N^{\circ} 3$

Análisis de ítems de la prueba de resiliencia

\begin{tabular}{cccccccc}
\hline $\mathrm{IT}$ & $\mathrm{r}$ & $\mathrm{IT}$ & $\mathrm{r}$ & $\mathrm{IT}$ & $\mathrm{r}$ & $\mathrm{IT}$ & $\mathrm{r}$ \\
\hline 01 & 0.3820 & 10 & 0.4824 & 19 & 0.3226 & 28 & 0.4621 \\
02 & 0.2130 & 11 & 0.5083 & 20 & 0.4026 & 29 & 0.2284 \\
03 & 0.2869 & 12 & 0.3851 & 21 & 0.2150 & 30 & 0.4534 \\
04 & 0.2860 & 13 & 0.4552 & 22 & 0.4313 & 31 & 0.4394 \\
05 & 0.2014 & 14 & 0.4731 & 23 & 0.3276 & 32 & 0.4862 \\
06 & 0.4774 & 15 & 0.3241 & 24 & 0.4259 & 33 & 0.3393 \\
07 & 0.3637 & 16 & 0.4489 & 25 & 0.2669 & 34 & 0.2177 \\
08 & 0.4151 & 17 & 0.4018 & 26 & 0.4295 & --- & ----- \\
09 & 0.3305 & 18 & 0.3695 & 27 & 0.4677 & --- & ---- \\
\hline
\end{tabular}


A continuación se procedió a calcular el coeficiente de confiabilidad por consistencia interna Alpha de Cronbach, cuyo resultado se presenta en la tabla 3.

Se puede observar que el coeficiente más bajo lo obtuvo la escala de interacción $(r=0.5225)$, mientras que el coeficiente más alto fue obtenido por la escala moralidad $(r=0.6734)$.

La prueba, en general, obtuvo un coeficiente de $0.8629(p<.001)$, el cual es altamente significativo, al igual que los demás, ello indica que el instrumento es consistente.

Tabla $\mathbf{N}^{\circ} 4$

Consistencia interna de la prueba de resiliencia

\begin{tabular}{lcc}
\hline Áreas & Alpha & $\mathrm{p}$ \\
\hline Insight & 0.5919 & 0.000 \\
Independencia & 0.5495 & 0.000 \\
Interaccion & 0.5225 & 0.000 \\
Moralidad & 0.6734 & 0.000 \\
Humor & 0.6410 & 0.000 \\
Iniciativa & 0.6475 & 0.000 \\
Creatividad & 0.6658 & 0.000 \\
Total & 0.8629 & 0.000 \\
\hline
\end{tabular}

\section{Procedimiento}

Se empleó el diseño de investigación descriptivo-comparativo. El método de estudio fue el descriptivo, haciendo uso de observaciones indirectas.

La técnica fue indirecta, ya que la recolección de información se hizo por medio de la Escala de Resiliencia para Adolescentes (ERA).
Para ejecutar la investigación se procedió a seleccionar tres establecimientos educativos típicos correspondientes a los niveles socioeconómicos alto, medio y bajo: Villa Caritas y San Pedro, del distrito de La Molina; Hans Christian Andersen, del distrito de Surco, y Fe y Alegría $\mathrm{N}^{\circ} 3$, del distrito de San Juan de Miraflores, que corresponden a los criterios de elección alto, medio y bajo, respectivamente.

Una vez identificada la muestra, correspondiente al segundo año de secundaria integrada por adolescentes entre 12 a 14 años, se aplicó la Escala de Resiliencia para Adolescentes, para proceder luego a la comparación respectiva, mediante el programa estadístico SSPS 9.0, en función de los objetivos considerados en la investigación,

\section{Resultados}

La tabla 5 describe los puntajes de la media y la desviación estándar para las variables de estudio, según el género.

Como se observa en la tabla 5, el género femenino obtiene mejores puntajes en la media en las áreas de: Insight, Interacción, Moralidad, Humor y Creatividad, mientras que el masculino lo obtiene en las áreas de Independencia e Iniciativa. 
Tabla $N^{\circ} 5$

Medias y desviaciones estándar

según género

\begin{tabular}{lrrrrrr}
\hline Áreas de resiliencia & \multicolumn{2}{c}{ Femenino } & \multicolumn{2}{c}{ Masculino } & \multicolumn{2}{c}{ Total } \\
\hline & Media & Ds St & Med & Des St & Med & Ds St \\
\hline Insight & 14.05 & 2.77 & 13.60 & 2.84 & 13.83 & 2.80 \\
Independencia & 8.84 & 1.96 & 9.73 & 2.56 & 9.27 & 2.31 \\
Interacción & 14.33 & 2.26 & 13.33 & 2.59 & 13.85 & 2.47 \\
Moralidad & 17.15 & 2.37 & 16.63 & 2.41 & 16.90 & 2.39 \\
Humor & 13.66 & 2.78 & 13.41 & 3.03 & 13.54 & 2.90 \\
Iniciativa & 14.45 & 2.51 & 14.63 & 2.09 & 14.54 & 2.31 \\
Creatividad & 11.19 & 2.89 & 10.39 & 2.64 & 10.80 & 2.79 \\
Total & 93.66 & 9.05 & 91.72 & 9.76 & 92.72 & 9.42 \\
\hline
\end{tabular}

Tabla $\mathbf{N}^{\circ} 6$

Medias y desviaciones estándar

según niveles socioeconómicos

\begin{tabular}{|c|c|c|c|c|c|c|c|c|}
\hline & \multicolumn{2}{|c|}{ Alta } & \multicolumn{2}{|c|}{ Media } & \multicolumn{2}{|c|}{ Baja } & \multicolumn{2}{|c|}{ Total } \\
\hline & Med & D.S & Med & D.S & Med & D.S & Med & D.S \\
\hline Insight & 13.6 & 2.30 & 13.15 & 2.68 & 14.40 & 3.08 & 13.83 & 2.80 \\
\hline Independencia & 9.19 & 2.35 & 9.11 & 2.30 & 9.43 & 2.31 & 9.27 & 2.31 \\
\hline Interacción & 13.05 & 2.14 & 13.85 & 2.59 & 14.34 & 2.48 & 13.85 & 2.47 \\
\hline Moralidad & 17.40 & 2.11 & 16.48 & 2.53 & 16.87 & 2.44 & 16.90 & 2.39 \\
\hline Humor & 12.95 & 2.71 & 14.26 & 3.02 & 13.42 & 2.87 & 13.54 & 2.90 \\
\hline Iniciativa & 15.17 & 2.41 & 14.72 & 2.38 & 14.01 & 2.10 & 14.54 & 2.31 \\
\hline Creatividad & 9.64 & 2.99 & 10.39 & 2.32 & 11.81 & 2.64 & 10.80 & 2.79 \\
\hline Total & 91.07 & 9.10 & 91.96 & 9.49 & 94.28 & 9.46 & 92.72 & 9.42 \\
\hline
\end{tabular}

La tabla 6 describe los puntajes de media y desviación estándar para las variables de estudio, según niveles socioeconómicos.

Como se observa en la tabla anterior, el nivel bajo obtiene mejores puntajes en la media en las áreas de Insight, Independencia, Interacción y Creatividad; el nivel alto obtiene mejores puntajes en Moralidad e Iniciativa, y el nivel medio en el área del Humor.
La tabla 7 presenta la prueba de Kolmogorov-Smirnov, para determinar si las variables de resiliencia presentan distribución normal.

Como se observa en la tabla 7, las áreas de Independencia, Moralidad, Humor e Iniciativa no tienen una distribución normal, ya que los coeficientes K-S son significativos $(\mathrm{p}<.05)$, y muy significativos $(\mathrm{p}<.01)$, por lo que se tomó la decisión de emplear estadística no paramétrica en todas las áreas. 
Tabla $N^{\circ} 7$

Prueba de Kolmogorov-Smirnov para determinar la distribución normal de las variables

\begin{tabular}{lll}
\hline $\begin{array}{l}\text { Áreas de } \\
\text { resiliencia }\end{array}$ & K-S & P \\
\hline Insight & 1.301 & $.068 \mathrm{n} . \mathrm{s}$ \\
Independencia & 1.839 & $.002^{*}$ \\
Interacción & 1.307 & $.066 \mathrm{n.s}$ \\
Moralidad & 1.780 & $.004^{*}$ \\
Humor & 1.553 & $.016^{*}$ \\
Iniciativa & 1.595 & $.012^{*}$ \\
Creatividad & 1.123 & $.161 \mathrm{n} . \mathrm{s}$ \\
Total & .0864 & $.444 \mathrm{n} . \mathrm{s}$ \\
\hline
\end{tabular}

* Significativo $(p<.05)$

n.s. no significativo (n.s. $>$.05)

Sin embargo, advertimos que el puntaje de resiliencia Total presenta una distribución normal $(\mathrm{K}-\mathrm{S}=.0864 ; \mathrm{P}=$ $.444)$, por lo que en este caso se utilizará estadística paramétrica.

Para analizar si existen diferencias significativas entre las áreas con respecto al género, se empleó la prueba no paramétrica "U" de Mann-Whitney, que es equivalente a la " $t$ " de Student.

A continuación, en la tabla 8 se observan los resultados de la prueba no paramétrica "U" de Mann Whitney para determinar los rangos medios de la variable de estudio según el género.
Tabla $N^{\circ} 8$

Rangos medios para la variable resiliencia según género

\begin{tabular}{lcc}
\hline $\begin{array}{l}\text { Áreas de } \\
\text { resiliencia }\end{array}$ & \multicolumn{2}{c}{ Rango medio } \\
Femenino & Masculino \\
\hline Insight & 80.04 & 75.83 \\
Independencia & 71.73 & 84.69 \\
Interacción & 86.79 & 68.62 \\
Moralidad & 82.74 & 72.94 \\
Humor & 79.66 & 76.23 \\
Iniciativa & 76.62 & 79.47 \\
Creatividad & 83.29 & 72.36 \\
\hline
\end{tabular}

La tabla 8 muestra que en las áreas de Interacción (86.79), Creatividad (83.29), Moralidad (82.74), Insight (80.04) y Humor (79.66) se observa un mejor desempeño a favor del género femenino, en comparación con el género masculino, el que tiene mejor desempeño en Independencia (84.69) e Iniciativa (79.47).

La tabla 9 presenta la prueba no paramétrica de U de Mann Whitney, para analizar si las diferencias entre las áreas con respecto a la variable género son significativas.

\section{Tabla $N^{\circ} 9$}

Prueba U de Mann Whitney para analizar diferencias en el género

\begin{tabular}{lcc}
\hline Áreas de resiliencia & "U" & $\mathrm{P}$ \\
\hline Insight & 2837.0 & .557 n.s \\
Independencia & 2498.5 & .069 n.s \\
Interacción & 2296.5 & $.011^{*}$ \\
Moralidad & 2620.5 & .170 n.s \\
Humor & 2867.0 & .632 n.s \\
Iniciativa & 2889.5 & .690 n.s \\
Creatividad & 2577.0 & .128 n.s \\
\hline
\end{tabular}

n.s Diferencias no significativas $(p>.05)$

* Diferencias significativas $(p<.05)$ 
Como se puede observar en la tabla anterior, sólo existe una diferencia significativa en el área de interacción $(\mathrm{U}=$ 2.296.5, $\mathrm{p}=.011$ ) a favor del género femenino.

La tabla 10 presenta los resultados de la prueba paramétrica t de Student, para determinar si existe diferencia significativa con respecto al puntaje total de resiliencia según el género.

\section{Tabla $\mathbf{N}^{\circ} 10$}

“t” de Student para el puntaje total de resiliencia según género

\begin{tabular}{lcccc}
\hline Resiliencia & Femenino & Masculino & $\mathrm{t}$ & $\mathrm{Sig}$ \\
\hline Total & 93.66 & 91.72 & 1.286 n.s & .200
\end{tabular}

En la tabla anterior se observa que no existen diferencias significativas respecto al género $(\mathrm{sig}=200, \mathrm{t}=1.286), \mathrm{a}$ pesar de que el género femenino tiene mayor puntaje en resiliencia que el masculino.

La tabla 10 presenta la prueba no paramétrica de Kruskal Wallis, para determinar los rangos medios del nivel socioeconómico de las áreas de la resiliencia.

Como se observa en la tabla 11, encontramos que el estrato bajo destaca en el área de Creatividad (94.00), Interacción (87.81), Insight (86.81) e Independencia (81.23). El estrato alto destaca en las áreas de Iniciativa (89.70) y Moralidad ( 87.06), y en el área de Humor (89.14) destaca el estrato medio.
Tabla No 11

Rangos medios para la variable resiliencia según nivel socioeconómico

\begin{tabular}{lccc}
\hline $\begin{array}{l}\text { Áreas de } \\
\text { resiliencia }\end{array}$ & Alto & Medio & Bajo \\
\hline Insight & 73.33 & 69.42 & 86.81 \\
Independencia & 75.77 & 75.33 & 81.23 \\
Interacción & 64.73 & 75.84 & 87.81 \\
Moralidad & 87.06 & 70.88 & 77.21 \\
Humor & 68.13 & 89.14 & 76.54 \\
Iniciativa & 89.70 & 82.03 & 67.90 \\
Creatividad & 59.95 & 71.17 & 94.00 \\
\hline
\end{tabular}

La tabla 12 presenta la prueba no paramétrica de Kruskal Wallis, para analizar si existen diferencias significativas en las áreas de resiliencia con respecto al nivel socioeconómico.

Tabla No 12

Prueba de Kruskal Wallis para analizar diferencias significativas respecto del nivel socioeconómico

\begin{tabular}{lrl}
\hline Areas & \multicolumn{1}{c}{$\mathrm{X}^{2}$} & $\mathrm{P}$ \\
\hline Insight & 4.782 & $.092 \mathrm{n} . \mathrm{s}$ \\
Independencia & .629 & $.730 \mathrm{n} . \mathrm{s}$ \\
Interacción & 7.088 & $.029^{*}$ \\
Moralidad & 2.943 & $.230 \mathrm{n} . \mathrm{s}$ \\
Humor & 5.002 & $.082 \mathrm{n} . \mathrm{s}$ \\
Iniciativa & 6.744 & $.034^{*}$ \\
Creatividad & 16.548 & $.000^{\star *}$ \\
\hline
\end{tabular}

n.s no significativo $(p>.05)$

* Significativo $(p<.05)$

${ }^{* *}$ Altamente significativo $(p<.001)$

Como se observa en la tabla anterior, se encontraron diferencias significativas en las áreas de interacción $(\mathrm{P}=$ .029) a favor del estrato bajo e iniciativa $(\mathrm{P}=.034)$ con respecto al nivel socioeconómico alto, observándose una diferencia altamente significativa en el 
área de creatividad $(\mathrm{p}=.000)$ a favor del estrato bajo.

En la tabla 13 se observan los resultados de la prueba "F" de análisis de varianza, para determinar si existe diferencia significativa en el puntaje de resiliencia respecto del nivel socioeconómico.

Tabla No 13

Prueba Anova para determinar diferencias significativas en resiliencia total respecto del nivel socioeconómico

\begin{tabular}{lcc}
\hline Resiliencia & $F$ & $P$ \\
\hline Total & 1.734 & 180
\end{tabular}

n.s diferencias no significativas $(p>0.05)$

Como se observa en la tabla 13 , no existen diferencias significativas con respecto al nivel socioeconómico en la resiliencia Total $(\mathrm{p}>.05)$.

\section{Discusión}

De los resultados obtenidos se colige que, respecto del puntaje total con relación al género, no existen diferencias significativas: $\mathrm{t}=1.286 \mathrm{n} . \mathrm{s}: \mathrm{Sig}=$ .200 , pero en cuanto a las variables encontramos que sí existen estas diferencias en el área de Interacción ( $\mathrm{U}=$ $2.539, \mathrm{p}>.011)$ a favor de las mujeres. El nivel de Interacción establece la capacidad de tener lazos íntimos y satisfactorios con otras personas, balanceando las propias necesidades con la simpatía y capacidad de dar a los otros. En los adolescentes es la necesidad de hacerse de apoyo social fuera de la fa- milia, vecinos, amigos u otros, que se preocupen por ellos; asimismo, es la capacidad de reclutamiento para estos fines. Esta área nos está demostrando que las adolescentes resilientes son aquellas que buscan soporte emocional en personas fuera de su entorno familiar, cuando éste no funciona o lo hace de manera disfuncional.

Sin embargo, es necesario establecer que el hecho de que no haya diferencias significativas en las demás áreas, nos permite establecer la presencia de los siguientes factores tanto para hombres como para mujeres adolescentes:

- Insight, es decir 1a habilidad para saber y entender las situaciones, verse diferentes de sus padres, no sentirse culpables.

- Independencia, definida como la capacidad para establecer límites entre los adolescentes y sus padres con problemas, manteniéndose física y emocionalmente distantes y no enganchar con la familia problema.

- Iniciativa, es decir, hacerse cargo de los problemas ejercitando control sobre ellos, estableciendo labores extraescolares, como practicar deporte y otros.

- Creatividad, considerada como la capacidad para imponer orden y belleza al caos de las experiencias problemas, moldearse en el esfuerzo y disciplina para convertir el caos en arte.

- Humor, orientado a encontrar lo cómico en la tragedia, usar el humor 
para transformar situaciones penosas y moralidad, considerada como la conciencia de tener una vida personal buena y satisfactoria para la humanidad, desarrollando valores propios de justicia y lealtad, enfrentar a los padres y adultos injustos, tratando de ser buenos.

Todos estos elementos son los que se convierten en recursos universales, para que los adolescentes puedan transformar el padecimiento, la desorganización y el caos familiar, los infortunios socioculturales de orden económico y otros, en factores que les permitan salir con éxito y desarrollarse con salud.

Se confirma la tesis de Rutter, en la que establece tres fuentes de resiliencia: las habilidades sociales en el manejo de situaciones, el ambiente facilitador y la fuerza psíquica interna. En nuestra investigación es altamente significativa para el género femenino la interacción, habilidad social en la que las mujeres tienen gran capacidad para tener lazos íntimos y satisfactorios con otras personas, balanceando sus propias necesidades, lo que ha sido corroborado en nuestra investigación en diferentes áreas.

Asimismo, confirmamos que la tesis de Krauskopf es válida para los adolescentes peruanos tomados en esta investigación, en el sentido de que la adversidad fortalece más que la no exposición al peligro, destacando que la sobreprotección vulnera la capacidad de enfrentar la adversidad. Desde este punto de vista, es válido afirmar que los sistemas de sobreprotección en nuestro país no son buenos para favorecer la resiliencia, por cuanto a los seres que los padecen no se les permite oportunidades para generar respuestas resilientes.

Burin, desde la teoría de la identificación de roles, propone la hipótesis de las identificaciones de modelos familiares, sobre todo de roles maternos. Al respecto, encontramos que los adolescentes investigados practican modelos identificatorios propios, buscando valores personales que se alejen de los modelos paternos, los que consideran perniciosos cuando la familia es disfuncional, encontrando que los adolescentes resilientes se establecen en función de modelos universales de humanidad, por ejemplo, en el área de la moralidad. Meler afirma que los modelos identificatorios desde la subjetividad se caracterizan por los vínculos derivados de la coexistencia de expectativas contradictorias, afirmando que es necesario establecer estudios culturales que instauren representatividades culturales. Nosotros afirmamos lo mismo en el sentido de que la cultura peruana provee a los adolescentes modelos familiares y culturales conflictivos y confusos, en algunos casos caóticos, como mecanismos identificatorios, encontrando en esta investigación que las adolescentes buscan modelos fuera 
del contexto familiar, pero que la cultura no ayuda a brindar modelos integrados para seguir; muy por el contrario, ofrece modelos pseudomutuales y escindidos, que perjudican los aspectos resilientes.

Respecto de lo que afirma Barbera, que el género es determinado por la vinculación sexo-género como una categorización normativa, nosotros encontramos que no es así, por cuanto no hemos hallado diferencias significativas para el género, excepto en el área de interacción a favor de las mujeres.

Respecto de los resultados de la resiliencia en las variables de nivel socioeconómico, encontramos que en términos globales no existen diferencias significativas en los valores totales ( $\mathrm{F}$ $=1.734 \mathrm{p}=.180$ ), esto significa que las clases sociales no son determinantes en la resiliencia. Grotberg encuentra lo mismo para los estudios realizados en la sociedad norteamericana, afirmando que los niveles socioeconómicos no están relacionados con la resiliencia. Baldwin, en sus estudios de niños y familias resistentes al estrés, afirma que la clase social es una importante variable de riesgo, sin embargo, establece diferencias entre la influencia de la clase social con las variables que él denomina proximales. En su investigación sostiene que la resistencia al estrés incide a través de las variables proximales, que están en relación con los factores de riesgo. Nosotros, en nuestra investigación, hemos encon- trado que si bien es cierto no hay diferencias significativas respecto de los valores totales, sí las hay respecto de algunas áreas, como Interacción ( $\mathrm{P}=$ .029) a favor a la clase baja, Iniciativa $(\mathrm{P}=.034)$, a favor de la clase alta, $\mathrm{y}$ Creatividad $(\mathrm{P}=.000)$ en valores altamente significativos a favor de la clase baja.

Es importante corroborar que en las clases sociales menos pudientes, determinadas como clase baja, el área de Creatividad es la más significativa, ya que implica la capacidad de poner orden al caos, encontrar belleza en medio de los problemas de las malas experiencias, y sobreponerse a los sentimientos de dolor. Éste es el motivo por el cual el adolescente de clase baja debe moldearse en un carácter de autoimposición, disciplina y esfuerzo para producir, teniendo en cuenta su condición de adolescente. Es justamente lo que se observamos en la conducta de los adolescentes resilientes, la necesidad de darle importancia al tiempo de vida y hacer productiva la existencia, sin detenerse en la depresión ni en las lamentaciones, buscando elementos motivadores para animarse a seguir y triunfar por encima de las dificultades. Debemos añadir que no sólo nos referimos a situaciones de índole socioeconómica, sino de pauperización generalizada, ya que en nuestro país las clases sociales están demarcadas por múltiples factores. Contreras afirma que la pobreza es explicada por causas indivi- 
duales, cuyas características son: bajo nivel educativo, inteligencia poco desarrollada, salud deficiente, discapacidad, marginalidad laboral, inequidad de oportunidades. Desde este punto de vista estamos de acuerdo con la tesis de Serrano, quien afirma que un enfoque de riesgo tomando el concepto de resiliencia, es sumamente importante por cuanto le brinda a los adolescentes capacidad de reacción e inmunidad, activando defensas protectoras como mecanismos personales a través de la familia $u$ organizaciones extrafamiliares.

Portocarrero establece diferencias conceptuales entre pobreza y exclusión social, afirmando que la pobreza no se refiere solamente a lo socioeconómico, sino más bien -y en ello coincidimosa dimensiones culturales de autoimagen y percepciones sobre carencias y posibilidades.

En cuanto a estos aspectos, en nuestra investigación encontramos que las áreas de Interacción $(\mathrm{P}=.029)$ y Creatividad $(\mathrm{P}=.000)$ marcan una significativa diferencia entre clases sociales, ya que la clase baja contrarresta sus deficiencias con capacidad de interacción con otras personas, balanceando sus propias necesidades con simpatía y capacidad de dar a otros, es decir, buscar una red de apoyo fuera del contexto familiar que se preocupe por ellos, lástima que en nuestra cultura lleve a la mendicidad como norma, al asistencialismo como mecanismo de control y no de ayuda.
Asimismo, se encuentra que la clase social baja usa la creatividad como un mecanismo resiliente por excelencia, utilizando esta capacidad para poner orden en el caos y en las experiencias problemas, mediante el esfuerzo y la disciplina.

Respecto de la variable Iniciativa $(\mathrm{P}=$ $.034)$, encontramos una diferencia significativa a favor de la clase alta, haciendo uso de este mecanismo para la solución de sus problemas, probándose a sí mismos frente a las labores demandantes, capacitándose en la tarea escolar, tratando de sobresalir en el deporte, realizando actividades extraescolares o buscando soluciones diversas, tomando como factor protector esta variable.

La presente investigación nos sirve, entonces, para comprender primero y desarrollar después, tareas urgentes en los niveles educativo, comunitario y clínico, favoreciendo las áreas de Iniciativa, Interacción y Creatividad, que hemos encontrado que marcan las diferencias entre las clases sociales, para establecer una planificación adecuada, que haga posible intervenir en forma urgente, con el fin de facilitar los aspectos resilientes como medidas psicológicas, que permitan al adolescente peruano mejorar su calidad de vida. 


\section{REFERENCIAS}

Aramburu, C. (1998). La revolución silenciosa. Cambios demográficos y sociales en los países andinos. En Desafios de la democracia en la región andina. Lima: Comisión Andina de Juristas.

Anthony, J. (1983). Las reacciones de los progenitores frente a los adolescentes y a su comportamiento. Parentalidad. Buenos Aires: Amorrortu.

Baldwin, A. (1982). Niños y familias resistentes al estrés. Proyecto de facilitación bibliográfica sobre resiliencia. Lima: Centro de Desarrollo y Asesoría Psicosocial (Cedapp).

Barbera, E. (1998). Psicología del género. Barcelona: Ariel S.A.

Burín, M. (1998). Género y familia. Buenos Aires: Paidós.

Contreras, V. (1998). Sembrando esperanzas. La superación de la pobreza mediante el desarrollo de la inteligencia en la infancia y la juventud. San José: Unicef.

Dolto, F. (1990). La causa de los adolescentes. Barcelona: Seix Barral.

Dryfoos, F.G. (1990). Adolescent at risk: Prevalence and prevention. New York: Oxford University Press.

Friedman, H.L. (1998). The health of adolescents: beliefs and behavior. Social science and medicine 305-315.

Grottberg. E. (1995). Guía de promoción de la resiliencia en los niños para fortalecer el espiritu humano. Washington: Organización Panamericana de la Salud.
Grotberg, E. (2000). Nuevas tendencias en resiliencia. Civitian International Research Center. UAB Washington University, School of Public Health, Services. Lima: Seminario Internacional sobre Resiliencia.

Horney, K. (1980). Psicología femenina. Madrid: Alianza.

Krauskopf, D. ( 1994). Adolescencia y educación. San José de Costa Rica: Euned. 2. ed.

Maddaleno, M. (1994). Riesgo en adolescentes. Presentado en el Curso de Multiplicadores de Salud Integrales de Adolescentes. Santiago: OPS Kellog.

Meler, I. (1992). Los roles de género en transición. Ponencia presentada en el VIII Congreso Metropolitano de Psicología. Buenos Aires: APBA.

Meler, I. \& Tajer, D. (2000). Psicoanálisis $y$ género. Debates en el foro. Buenos Aires: Lugar Editorial.

Obiols \& Di Segni de Obiols (1994). Adolescencia, postmodernidad y escuela secundaria. Buenos Aires: Kapelusz.

Portocarrero, F. (2000). Políticas sociales en el Perú. Nuevos aportes. Lima: Universidad del Pacífico. Centro de Investigación.

Prado, R. \& Del Águila, M. (2000). Estructura y funcionamiento familiar en adolescentes resilientes y no resilientes. Lima: Investigación realizada en la Facultad de Psicología de la Universidad Federico Villarreal.

Rutter, M. (1992). Resilience: Some conceptual considerations. Conference on social change and focus on the Americas. 
Serrano, C.V. (1995). La salud del adolescente y el joven. La salud integral de los adolescentes y los jóvenes: su promoción y su cuidado. Washington: Organización Panamericana de la Salud (Publicación $\mathrm{N}^{\circ}$ 552).

Werner and Smith (1982). Vulnerable but invencible. A longitudinal study of resilient children and youth. New York: Mac Graw-Hill.

Wolin, S. \& Wolin (1993). The resilient self: How survival of troubled families rise above adversity. Washington: Villard Books. 